Revista Jurídica de la Universidad de León, núm. 5, 2018, pp. 65-82

\title{
LE CONSEGUENZE DEI VIZI DI FORMA E PROCEDURA NEI LICENZIAMENTI INDIVIDUALI E COLLETTIVI/LAS CONSECUENCIAS DE LOS VICIOS DE FORMA Y PROCEDIMIENTO EN EL DESPIDO INDIVIDUAL Y COLECTIVO
}

\author{
Marco Mocella \\ marco.mocella@unisannio.it \\ Ric erc atore nell' Università deg li Studi del Sa nnio
}

Rec ibido: 05/06/2018 - Aceptado: 31/10/2018

\section{Sommario}

L'autore analizza, in una prospettiva evolutiva, il vincolo di forma nei licenziamenti. Vengono in primo luogo esaminate le conseguenze della mancanza di forma scritta del licenziamento per distinguerla dalla mancata indicazione dei motivi, oggi distinti anche sotto il profilo

- Parole chiave: Licenziamenti individuali e collettivi; Obblighi di forma e procedura; Sanzioni; Modifiche 2012 e 2015 sanzionatorio come ipotizzato in passato da attenta dottrina. Esaminate quindi le procedure previste per il licenziamento per giustificato motivo oggettivo e giusta causa, l'autore verifica la procedura dei licenziamenti collettivi di matrice europea e le modificazioni introdotte dalle riforme del 2012 e del 2015, evidenziando le problematiche connesse al depotenziamento dei vincoli formali.

Abstract

- Keywords:

Individual and collective redundancies; Formal and procedures obligations; Penalties; Italian law; Changes 2012 and 2015
The author analyzes, in an evolutionary perspective, the obligation of the form in dismissals. Firstly, the consequences of the absence of a written form of dismissal are examined to distinguish them from the lack of reasons indication, which are presently distinct from the point of view of sanctions, as it has been hypothesized in the past by careful interpretation. After examining the procedures for dismissals for economic and disciplinary reasons, the author checks the procedure of collective redundancies of European origin, and the changes introduced by the reforms of 2012 and 2015, highlighting the problems related to the weakening of the form.

\section{Resumen}

El autor analiza, en una perspectiva evolutiva, la restricción de la forma en los despidos. En primer lugar, se examinan las consecuencias de la falta de forma escrita del despido para distinguirlo de la falta de indicación de las razones, que hoy se distinguen por la sanción tal como fue hipotizada en el pasado por la
- Palabras clave:

Despidos individuales y colectivos; Requisitos de forma y procedimiento; Sanciones; Ley italiana; Modificaciones 2012 y 2015 
doctrina. Después de examinar los procedimientos para el despido por razones objetivas justificadas y justa causa, el autor verifica el procedimiento de despidos colectivos de la matriz europea y los cambios introducidos por las reformas de 2012 y 2015, destacando los problemas relacionados con el debilitamiento de las limitaciones formales.

1. L'introduzione dell'obbligo della forma scritta del licenziamento nell'ordinamento italiano: individuazione delle conseguenze e inefficacia del recesso - 2. L'obbligo di comunicazione dei motivi - 3. La procedura per il licenziamento disciplinare e per giustificato motivo oggettivo -4 . Le conseguenze delle violazioni della procedura prevista per i licenziamenti collettivi -5 . Conclusioni

\section{L'INTRODUZIONE DELL'OBBLIGO DELLA FORMA SCRITTA DEL LICENZIAMENTO NELL'ORDINAMENTO ITALIANO}

La disciplina degli obblighi formali connessi al recesso ha subito sostanziali modifiche ad opera delle recenti riforme dei licenziamenti, in particolare, la 1. 28/6/2012 n.92 e il d.lgs. 4 marzo 2015 n. 23.

Per comprendere appieno la portata delle nuove norme, tuttavia, è necessario premettere una breve ricognizione degli obblighi di forma e procedura relativi ai licenziamenti, con particolare riguardo all'importante funzione svolta da dottrina e giurisprudenza nell'esegesi, spesso creativa, di tali regole.

Originariamente, l'ordinamento italiano non prevedeva nessun obbligo relativamente alla forma e alla procedura dei licenziamenti che, pertanto, potevano essere intimati anche oralmente, senza alcuna formalità. La prima previsione di un obbligo di forma e motivazione del licenziamento individuale venne introdotta con la legge 15 luglio 1966 n. 604, mentre la successiva 1. 11/5/90 n.108 ne estese l'ambito applicativo a tutti i datori di lavoro; la 1. 23/7/91 n.223 ha previsto poi anche per i licenziamenti collettivi la necessità di una forma e procedura vincolata, in attuazione (invero assai tardiva) della direttiva 129/75/CE.

Peraltro, come per i licenziamenti collettivi, anche per quelli individuali forma e procedura erano già state introdotte dalla contrattazione collettiva (cfr. accordi interconfederali del 1950 e del 1965) di cui la legge recepì sostanzialmente i contenuti.

L'estensione operata dal secondo comma dell'art. 2 della 1. 11/5/90 n.108 della comunicazione per iscritto del licenziamento a tutti i datori di lavoro, con pochissime eccezioni $^{1}$, concluse infine un processo interpretativo, iniziato in dottrina $\mathrm{e}$

1 Le uniche eccezioni all'obbligo di forma scritta sono quelle relative ai datori di lavoro domestici (art. 4, comma 1,1. 108/1990, che esclude i "rapporti disciplinati dalla legge 2 aprile 1958 n. 339"), in ragione della peculiarità del rapporto, e ai prestatori di lavoro ultra sessantenni aventi $\mathrm{i}$ requisiti pensionistici, sempre che non abbiano optato per la prosecuzione del rapporto (art. 4, comma 2, 1. 108/1990), gli sportivi professionisti nonché $\mathrm{i}$ lavoratori in prova, esclusi ex art. 10 L.604/66 dall'intera legge (art. 10, 1. 604/1966). Finanche per i dirigenti, (art. 2, comma 4, 1. 604/1966), il licenziamento deve essere 
giurisprudenza subito dopo l'emanazione della 1. 15/7/66 n.604, tendente a generalizzare tale obbligo ${ }^{2}$. Alla luce del nuovo ambito applicativo generale della forma scritta, il licenziamento finisce col divenire un atto a forma vincolata ad substantiam ${ }^{3}$.

La ratio dell'imposizione della forma scritta per il recesso è stata ricondotta da un lato all'esigenza di certezza del diritto, quindi alla garanzia dell'esistenza stessa dell'atto a tutela del lavoratore da arbitrii del datore di lavoro, che in taluni casi poteva negare addirittura il recesso ${ }^{4}$; dall'altro, alla necessità di imporre una particolare attenzione nella fase normalmente più traumatica del rapporto, i cui effetti travalicano l'aspetto lavorativo 5 .

intimato per iscritto ancorché senza necessità di alcuna motivazione. F. MAZZıOTTI, "Forma e procedura dei licenziamenti" in F. CARINCI, La disciplina dei licenziamenti dopo le leggi 108/1990 e 223/1991, Napoli 1991, 71; M. ZiLIOTTI, "Commento all'art.2" in L. Galantino, La disciplina dei licenziamenti individuali e collettivi, Torino, 1993, 22.

2 Prima dell'entrata in vigore della L. 108/90, l'obbligo di forma previsto dall'art. 2 L. 604/66 riguardava solo i datori di lavoro con più di 35 dipendenti, ex art. $11,1^{\circ}$ comma. A seguito dell'emanazione della L. 300/70, art. 18, l'obbligo di forma era stato ritenuto applicabile anche alle imprese industriali o commerciali, naturalmente nelle unità produttive con più di 15 dipendenti. Cfr. Corte Cost. 6/3/74 n ${ }^{\circ} 55$ in Foro It., 1974, I, 959 con nota di G. PerA; M.V. Ballestrero, I licenziamenti, Milano, 1975, 276; P. Genoviva, I licenziamenti, Torino 1988, 20; Roccella M., "I licenziamenti nella piccola impresa" in Dir. Prat. Lav., 1990, 666.

3 Sulla forma come garanzia per il prestatore P. PERLINGIERI, Forma degli atti e formalismo degli interpreti, Napoli, 1987, 80ss; ID., "La forma legale del licenziamento individuale come "garanzia"' in Rass. Dir. Civ., 1986, 1071, ed in particolare la nota 19; M. D'ONGHIA, La forma vincolata nel diritto del lavoro, Milano, 2005, 85ss; G. Ferraro, "Formalismo giuridico e diritto del lavoro", in Dir. Lav. Rel. Ind., 1989, 555. Più di recente, M. D'ONGHiA, V. BAVARO, "Profili costituzionali del licenziamento nullo" in WP C.S.D.L.E. “Massimo D'Antona”.'IT, 2016, 305, 17ss.

4 P. Perlingieri, Forma degli atti e formalismo degli interpreti, cit., 84; G. Ferraro, Formalismo giuridico e diritto del lavoro cit., 544. Sull'esigenza di certezza del diritto anche per il datore di lavoro, sia per ragioni legate all'individuazione corretta della modalità risolutiva del rapporto sia oggi per la possibilità offerta allo stesso di revocare il licenziamento v. infra. Corte Cost. 6/3/74 n. 55 cit.; M.V. BALleSTRERO, I licenziamenti, cit., 276; Genoviva, I licenziamenti, Torino 1988, 20; M. RocCELla, "I licenziamenti nella piccola impresa" in Dir. Prat. Lav., 1990, 666; NERI BERNARDI, "I licenziamenti individuali e indennità risarcitoria in caso di tutela obbligatoria del posto di lavoro" in Riv. Trim. Dir. Proc. Civ., 1993, 917; NicCOlaI, "Gli effetti del licenziamento individuale illegittimo" in Lav. Dir., 1982, 119; P. Perlingieri, Forma degli atti e formalismo degli interpreti, cit., 84; G. FerRARO, Formalismo giuridico e diritto del lavoro cit., 544. In giurisprudenza Cass. 29/1/85 n. 519, in Giust. Civ. 1985, I, 2788.

5 Corte Cost. 23/11/94 n³98 in Resp. Civ. Prev., 1995, 516 con nota L. Nogler; in Riv. It. Dir. Lav., 1995, II, 3 con nota di G. PerA; in Riv. Crit. Dir. Lav., 1995, 249 con nota di F. Amato; in Riv. Giur. Lav., 1995, II, 443 con nota di Di FranCESCO; in Giust. Civ., 1995, 571 con nota di SCARTOZZI; in Giur. It., 1995, I, 471 con nota di Di FranCESCO; in Dir. Lav., 1995, II, 253 con nota di BAtTiSTA; in Dir. Lav., 1994, II, 507 con nota di M. Mocella; in Giust.Civ., 1995, I, 2757 con nota di NANNIPIERI; Foro It., 1995, I, 9. Corte Cost. 7/7/86 n ${ }^{\circ} 176$ in RassDC, 1986, 1069 ed in Giust.Civ., 1986, 1368. 
Va subito notato che tale obbligo non ha subito alcuna modifica sostanziale dalle riforme del 2012 e 2015 ed è quindi rimasto sostanzialmente immutato.

Con riguardo alle conseguenze della mancanza di forma scritta del licenziamento, occorre evidenziare che, anteriormente alle riforme suddette, in caso di mancanza di forma scritta dell'atto di licenziamento, qualora il datore fosse stato soggetto al regime di stabilità reale si applicava pacificamente quest'ultimo ${ }^{6}$. Negli altri casi, la prevalente dottrina e giurisprudenza riteneva dovesse applicarsi la cosiddetta tutela reale di diritto comune: il licenziamento era considerato inefficace non producendo quindi alcun effetto sulla continuità del rapporto che doveva considerarsi come mai interrotto ${ }^{7}$. Sebbene in questo modo si cercasse di estendere ai lavoratori cui non fosse applicabile l'art. 18 L. 300/70 una tutela quasi equivalente ${ }^{8}$, le differenze tra le due ipotesi non erano trascurabili: infatti la tutela reale di diritto comune non era soggetta al risarcimento minimo delle 5 mensilità previsto dall'art. 18 Statuto dei lavoratori (1. 20/5/70 n.300) né alle decurtazioni dell'aliunde perceptum e il lavoratore non aveva la facoltà di optare per l'indennità alternativa alla reintegra pari a 15 mensilità di cui all'art. 18 Statuto dei lavoratori ${ }^{9}$.

6 P. Genoviva, op. cit., 83; M. PaPaleoni, La fine del libero licenziamento, Milano, 1991, 229; E. BALLETTI, "Riassunzione o risarcimento del danno" in I licenziamenti individuali a cura di G. Ferraro, Napoli 1990, 95; R. De Luca TAmajo, "Disciplina dei licenziamenti individuali" in Le nuove leggi civili commentate, a cura di R. DE LuCA TAMAJO, M. D'Antona, 1991, 187; M. ZiLiotTi, op. cit., 26. Corte Cost. 22/1/87 n. 17, in Giur. Cost., 1987, I, 118; in Riv. It. Dir. Lav., 1987, II, 431; in Dir.Lav., 1987, II, 130.

7 Per la tesi della nullità di diritto comune P. TulLINI, "La cd. tutela reale di diritto comune", in Quad. Dir. Lav. Rel. Ind., n. 8, 1990, 103 ss. In giurisprudenza, Cass. 22/1/91 n ${ }^{\circ} 542$ in Foro It., 1992, 1142 con nota di De LuCA; Cass. 3/6/92 n6741 in Giust. Civ., 1993, I, 81 con nota di M. PAPALEONi; Cass. 4/3/92 n²596 ivi, 1993, I, 840 con nota di ProIETTI; Cass. 8/7/88 n²521, in Foro It., 1988, I, 3592; Cass. 28/10/97 n 10624 in Guida Dir., 1997, 44, 72 (per gli obblighi contributivi), Cass. 20/2/99 n 1444 in Guida Lav., 1999, 14, 54. Questa interpretazione è stata quindi avallata da Cass. S.U. 27/7/99 n508 in Guida Dir., 1999, 32, 30. Per l'applicazione della tutela di diritto comune in ipotesi di nullità diverse dal licenziamento orale Cass.4/2/92 n ${ }^{\circ} 1159$ in Giust. Civ., 1992, I, 2123 (sul licenziamento per causa di matrimonio); Cass. 9/9/95 n ${ }^{\circ} 9549$ in Not. Giur. Lav., 1995, 769 (sul licenziamento nel periodo di interdizione). Una autorevole ma minoritaria corrente di pensiero propendeva invece per l'applicazione dell'art. 8 dellal.604/66: Cass.23/11/92 n²12486 in Riv. It. Dir. Lav., 1993, II, 355; Trib. Milano 9/2/94 in Lav. Giur., 1994, 913 con nota di D'AvossA. In dottrina G. Pera, Le novità nella disciplina dei licenziamenti, Padova, 1993, 68 ss; M.PAPALEONI, "Licenziamento disciplinare e nullità di diritto comune" in Riv. It. Dir. Lav., 1995, II, 653; MARINO, "Il vizio di forma del licenziamento viziato nella procedura: una svolta di rilievo", in Riv. It. Dir. Lav., 1993, II, 357ss; G. PERA, Ancora sul vizio di forma nella zona della tutela debole contro il licenziamento in Giust. Civ., 1997, I, 938. Sul punto Corte Cost 23/11/94 n³98 cit.; Cass. 20/5/94 n4938 in Rep. Foro It., voce Lavoro (rapporto), 1994, 1511.

8 Cass. 3/1/86 n²3 in Dir. Prat. Lav., 1986, 1029; Cass. 18/10/82 n 5394 in Not. Giur. Lav., 1982, 906; Cass. 1/8/91 n8483, ivi, 1991, 243.

9 R. De Luca Tamajo, Disciplina dei licenziamenti individuali cit., 184. La decurtazione dell'aliundem perceptum viene da alcuni autori considerata possibile nella tutela reale di diritto comune e non nel caso di applicazione dell'art. 18 Statuto, dopo la novella dell'art. 1 L.108/90. 
Tali aspetti sono stati risolti con la riforma Fornero (1. 28/6/2012 n.92) che, nel riscrivere il nuovo testo art. 18 , c. 1,1 . n. 20/5/70 n.300, ha esteso la tutela reintegratoria a tutti i datori indipendentemente dal numero dei dipendenti occupati, comprese quindi le piccole imprese.

Analogamente, il d. lgs. 4 marzo 2015, n. 23, in attuazione della legge 10 dicembre 2014, n. 183, recante disposizioni in materia di contratto di lavoro a tempo indeterminato a tutele crescenti (cd. Jobs Act), non ha sostanzialmente modificato la disciplina riproponendo quanto previsto sul punto dalla 1. 28/6/2012 n. $92^{10}$.

In breve, entrambe le normative prevedono la nullità del licenziamento con conseguente condanna del datore di lavoro al risarcimento del danno subito dal lavoratore commisurato all'ultima retribuzione di riferimento per il calcolo del trattamento di fine rapporto dal giorno del licenziamento sino a quello dell'effettiva reintegrazione, dedotto quanto percepito per lo svolgimento di altre attività lavorative. Restano immutati l'ammontare minimo del risarcimento, che non potrà essere inferiore a cinque mensilità oltre al versamento dei contributi previdenziali $\mathrm{e}$ assistenziali, e la facoltà del lavoratore di richiedere l'indennità in sostituzione della reintegrazione in misura pari a quindici mensilità non assoggettata a contribuzione previdenziale.

\section{L'OBBLIGO DI COMUNICAZIONE DEI MOTIVI}

Con riguardo alla comunicazione dei motivi del licenziamento, prima del 2012 essa non era obbligatoria per il datore di lavoro a meno che non venisse richiesta dal

10 F. CARINCI, C. CESTER. (a cura di), Il licenziamento all'indomani del d.lgs. n. 23/2015 (contratto di lavoro a tempo indeterminato a tutele crescenti), Modena, 2015; E. GHERA, D. GAROFALO (a cura di), Le tutele per i licenziamenti e per la disoccupazione involontaria nel Jobs Act 2, Bari, 2015; L. FIorillo, A. Perulli, Contratto a tutele crescenti e Naspi, Torino 2015; R. Pessi, C. Pisani, G.Proia, A. Vallebona (a cura di), Jobs Act e licenziamento, Torino, 2015; AA.VV., La riforma del lavoro (c.d. Jobs Act). Il contratto di lavoro a tutele crescenti e gli strumenti di contrasto alla disoccupazione (d.leg. 4 marzo 2015 nn. 22 e 23), in Foro It., 2015, V, col. 229; G. ZiLIO Grandi, M. Biasi (a cura di), Commentario breve alla riforma "Jobs act", Padova, 2016; F. CARINCI, "Un contratto alla ricerca di una sua identità: il contratto a tempo indeterminato a tutele crescenti (ai sensi della bozza del decreto legislativo 24 dicembre 2014)", in F. CARINCI, M. TIRABOSCHI (a cura di), I decreti attuativi del Jobs Act: prima lettura e interpretazioni, Modena, 2015, 65ss; ID., Il tramonto dello Statuto dei lavoratori (dalla L. N. 300/1970 al Jobs Act), in ADAPT LABOUR STUDIES e-Book series, n. 41/2015; L. NOGLER, "I licenziamenti per giustificato motivo soggettivo o per giusta causa nel d.lgs. n. 23 del 2015", in Arg. Dir. Lav., 2015, 3, 507ss.; R. DE LUCA TAMAJO, "Licenziamento disciplinare, clausole elastiche, "fatto" contestato", ivi, 2, 269ss.; M. MARAZZA, "Il regime sanzionatorio dei licenziamenti nel Jobs Act", in Arg. Dir. Lav., 2015, 2, 310ss.; M. PERSIANI, "Noterelle su due problemi di interpretazione della nuova disciplina dei licenziamenti", Arg. Dir. Lav., 2015, 393ss; G. VIDIRI, "Il licenziamento disciplinare nel primo decreto attuativo del Jobs Act tra luci e (non poche) ombre", in Arg. Dir. Lav., 2015, 353ss.; P. ICHINO, "La riforma del lavoro in Italia. Una nuova cultura delle relazioni industriali”, in Riv. It. Dir. Lav., 2015, I, 205 ss. 
lavoratore nei termini di legge, nel cui caso il datore di lavoro doveva di comunicare per iscritto entro sette giorni le motivazioni alla base del recesso.

L'art. 2 secondo comma della legge 604/66 era stato modificato dalla legge 108/90 solo per i termini per la richiesta e la comunicazione dei motivi, mentre la proposta di unificare la comunicazione del recesso con quella dei motivi non era stata, invece, accolta dal legislatore in quella sede; anche la comunicazione dei motivi doveva certamente rivestire la forma scritta ${ }^{11}$.

Lo scopo di tale obbligo era stato identificato da dottrina e giurisprudenza nell'esigenza di garantire al lavoratore la possibilità di conoscere i fatti posti a fondamento del recesso, onde poter valutare se impugnarlo o meno, per cui essa doveva avvenire non solo tempestivamente ma anche in forma analitica e completa ${ }^{12}$.

La mancata comunicazione dei motivi o anche la sua tardività ovvero incompletezza era sanzionata dal legislatore analogamente alla mancata comunicazione del licenziamento, quindi con l'inefficacia dell'atto ${ }^{13}$.

A seguito della riforma della 1. 92/2012, viene operata una differenziazione tra nullità ed inefficacia del licenziamento, sul presupposto che l'inefficacia in senso stretto si avrebbe solo quando difetti un elemento esterno all'atto stesso, come nel caso dell'omessa comunicazione dei motivi ${ }^{14}$; diversamente, in caso di mancanza della forma l'atto sarebbe nullo ${ }^{15}$.

11 Sulla nullità del licenziamento in caso di comunicazione orale dei motivi Cfr. Cass. S.U. 18/10/82 n5394 Foro It., 1983, I, 2206; Cass. 3/11/77 n4687 in Riv. Giur. Lav, 1978, II, 939; E. BALLETTI, "Riassunzione o risarcimento del danno" in I licenziamenti individuali a cura di Ferraro, Napoli 1990, 94; ZiliotTI, "La forma del licenziamento" in Commento alla l.604/66 a cura di GALANTINO, Torino 1990, 21.

12 Cass. 21/11/89 n ${ }^{\circ} 5040$ in Not. Giur. Lav., 1990, 403. In dottrina M. ZiLIOTTI, op. cit., 21; F. MazziotTi, Forma e procedura dei licenziamenti cit., 76.

13 Cass. 23/12/96 n 11497 in Guida Dir., 1997, 12, 36 con nota adesiva di A. PelaGgI secondo cui occorrerebbe distinguere tra inefficacia derivante da mancanza di forma scritta e inefficacia derivante da omessa o tardiva comunicazione dei motivi. In dottrina A. Di MAJO, "I licenziamenti illegittimi tra diritto comune e diritto speciale" in Riv. Giur. Lav., 1974, I, 272; M.V. Ballestrero, I licenziamenti, cit., 104; U. Romagnoli, "Sub. art. 18" in Ghezzi, Mancini, Montuschi, Romagnoli, Statuto dei diritti dei lavoratori, Bologna Roma, 1972, 95; P. Tullini, op. cit., 104; G. PERA, La cessazione del rapporto di lavoro, in Enc. Giur. Lav. a cura di MAZzoni, V, Padova 1980, 178.

14 F. Mazziotti, "Licenziamenti illegittimi e provvedimenti giudiziari", in Atti delle giornate di studio di diritto del lavoro, Torino, 16-17 maggio 1987, Milano, 1988, 162. Cass. S.U. 2/3/87 n. 2180, in Giust. Civ., 1986, I, 1055; Cass. 28/10/89 n4542, in Not. Giur. Lav.., 1989, 706; in Orient. Giur. Lav., 1990, 154; Cass. 1/8/91 n. 8483, in Dir. Prat. Lav., 1991, 2793; Cass. S.U. 21/2/84 n. 1236, in Foro It., 1984, 2552; in Giust. Civ., 1984, I, 1784 con nota di PAPAleOni; in Riv. It. Dir. Lav.., 1984, II, 682 con nota di Orsi Vergiati; in Not. Giur. Lav., 1984, 277; A. TAZZI, Sul licenziamento disciplinare nelle piccole imprese in Dir. Lav., 1993, II, 434.

15 F. MAZZIOTTI, Forma e procedura dei licenziamenti cit., 72; G. NAPOLETANO, La nuova disciplina dei licenziamenti individuali, Napoli 1990, 97; M.V. BALlestrero, I licenziamenti, Milano 1975, 95; G. GianPICCOLO, "Dichiarazione recettizia", in 
Pertanto, mentre la mancanza di forma del licenziamento continua ad essere sanzionata con l'inefficacia del recesso, l'omissione della comunicazione dei motivi, che ora devono essere inclusi nella comunicazione del licenziamento, viene colpita da una sanzione conservativa assai più lieve, da un minimo di sei ad un massimo di dodici mensilità (art. 18 comma sesto). Si assiste, dunque, ad un notevole capovolgimento di prospettiva che si riflette, evidentemente, anche sulla ratio sottesa alle prescrizioni formali: da un lato queste vengono ampliate, dall'altro si distingue nettamente tra la mancanza di forma dell'atto e l'omissione o irregolarità delle ulteriori formalità imposte dalla legge.

Tale differenziazione finisce con l'affermarsi ancor più nettamente a seguito della riforma del 2015, in quanto, nell'ipotesi in cui il recesso sia intimato per iscritto ma con violazione del requisito di motivazione di cui all'articolo 2 , comma 2 , della legge n. 604 del 1966 o della procedura del licenziamento disciplinare di cui all'articolo 7 della legge n. 300 del 1970, il rapporto di lavoro si estingue comunque ed il datore di lavoro può essere condannato esclusivamente al pagamento di un'indennità pari ad una mensilità per ogni anno di servizio, in misura comunque non inferiore a due e non superiore a dodici mensilità; peraltro, nell'art. 4 d.lgs. 23/2015 il Legislatore non utilizza più, condivisibilmente, il termine "inefficacia".

La declinazione di una diversa tipologia sanzionatoria riflette una diversa funzione della prescrizione formale nelle diverse ipotesi esaminate e, in quest'ottica, la scelta del Legislatore di valorizzare una funzione rispetto all'altra risponde ad una logica che sembrerebbe non contrastare con alcuna norma costituzionale.

Il depotenziamento delle sanzioni formali diviene invece maggiormente penalizzante per il prestatore con riferimento ai vizi relativi alla comunicazione dei motivi del recesso e, in parte, alla procedura per i licenziamenti disciplinari (infra). Nel primo caso, infatti, si rende estremamente difficoltoso per il prestatore individuare le ragioni che hanno portato alla scelta di recedere dal rapporto e quindi valutare eventuali profili che potrebbero rendere l'atto nullo; nel secondo, in quanto dalla inesistenza del fatto può ancora derivare la sanzione reintegratoria, per cui la conoscenza esatta dell'addebito diviene essenziale per valutare l'esistenza o meno dello stesso.

Non sembra revocabile in dubbio che la scelta di ridurre le conseguenze previste per tale violazione si ponga in contrasto con il ruolo attribuito alla comunicazione dei motivi nel licenziamento individuale, vale a dire l'esigenza di garantire al lavoratore di avere piena conoscenza dei fatti posti a fondamento del recesso, allo scopo di porlo in condizione di valutare scientemente se impugnare o meno l'atto e consentirgli

Enciclopedia del diritto, XII, Milano 1964, 389; M. GiongiannI, "Forma degli atti (diritto privato)",in Enciclopedia del diritto, XVII, 1968, Milano 1968, 993; A. LISERRE, "Forma degli atti (diritto civile)", in Enciclopedia del diritto, XIV, 1989; G.B. FERRI, "Forma e contenuto degli atti giuridici", in Riv. Dir. Comm., 1990, I, 1; V. SCALISI, "Inefficacia" in Enciclopedia del diritto, 1971, 325; G. MiRABELLI, "Dei contratti in generale" in Commentario Codice civile, Torino 1958, 371; SACCo R., Il contratto, Torino 1993. 
quindi un pieno esercizio di difesa nel momento in cui intenda effettivamente agire in giudizio $^{16}$.

Inoltre, sebbene resti fermo l'obbligo del datore di lavoro di comunicare in maniera analitica e completa la motivazione, un'eventuale incompletezza della motivazione non potrebbe ovviamente essere sanzionata in misura più grave dalla sua mancanza. Dunque, in caso di motivazione insufficiente, si dovrebbe ritenere questa del tutto inesistente, con applicazione della relativa nuova sanzione.

\section{LA PROCEDURA PER IL LICENZIAMENTO DISCIPLINARE E PER GIUSTIFICATO MOTIVO OGGETTIVO}

Il procedimento disciplinare in Italia è previsto dall'art. 7 dello Statuto dei Lavoratori (1. 20 maggio 1970 n.300) che ai primi tre commi dell'art. 7 della L. 300/70 regola in generale il procedimento disciplinare valido per tutte le sanzioni, compresi i licenziamenti disciplinari, come stabilito in via interpretativa dalla Corte Costituzionale $^{17}$ e da quella di Cassazione ${ }^{18}$.

La prima aveva inoltre chiarito che, in caso di violazione della procedura dell'art. 7, l'infrazione disciplinare non poteva essere posta a giustificazione del licenziamento, il quale era quindi sanzionato in ragione dei requisiti dimensionali ${ }^{19}$.

16 Per il licenziamento ingiurioso, peraltro, già in precedenza si riteneva che la comunicazione non potesse essere contestuale al recesso; l'interesse del lavoratore sarebbe stato quindi comunque salvaguardato: Cass. 21/6/79 n 3488 in Giur. It., 1980, I, 1, 505; Cass. 22/1/66 $\mathrm{n}^{\circ} 557$ in Foro It., 1966, I, 1762. In dottrina MAZZIOTTI F., Forma e procedura, cit., 73; M. ZiLIOTTI, op. cit., 21. Per la risarcibilità del danno nel caso di comunicazione di motivazione ingiuriosa Cass. 22/1/66 n॰557 cit.; Cass. 29/4/81 n²637 Foro It., 1981, I, 1557. Sulla mancata contestazione dell'addebito disciplinare v. però Cass. 6/11/14 n. 23669 in Arg. Dir. Lav., 2014, 6, 1381; Lav. Giur., 2015, 2, 152 nota di BuCONI; in Foro It., 2014, 12, 1 , 3418 GI, 2014, 12, 2788 nota di FIORILLO.

17 Corte Cost. 30/11/82 n.204542, Foro It., 1982, I, 2981. Con la sentenza n ${ }^{\circ} 427 / 89$ la Corte aveva poi esteso le garanzie procedimentali dell'art. 7 anche ai datori di lavoro non assoggettati alle norme limitatrici dei licenziamenti

18 Cfr. Cass. 27/10/83 n ${ }^{\circ} 6356$ in Foro It., Rep., 1983, voce Lavoro, (Rapporto), 2328; Cass. 7/11/83 n 6579, ivi, 1984, I, 97; Cass. 25/7/90 n 7520, ivi, Rep., 1990, voce cit., 1788; Cass. 27/11/92 n 12666, ivi, Rep. 1992 voce cit., 1689; Cass. 27/1/93 n 1000, ivi, Rep 1993, voce cit., 1394.

19 Cass. $n^{\circ}$ 5262/88 in Foro It., Rep. 1988, Lavoro (rapporto), 1979. In dottrina F. FocARETA, "L'articolo 18 St. lav. fra tendenze espansive e problemi irrisolti", in Quad. Dir. Lav. Rel. Ind., 1989, 6, 269; R. De LuCA TAMAJo, F. BianChi D'Urso, "Licenziamento disciplinare e Corte costituzionale", in Not. Giur. Lav., 1989, 148. Contra, per la tesi dell'applicazione espansiva dell'art. 18 a tutte le ipotesi di licenziamento invalido, compreso quello per violazione dell'art. 7 St.: M. G. GAROFALO, "La nuova disciplina dei licenziamenti individuali: prime osservazioni", in Riv. Giur. Lav., 1990, I, 184; V. CERRITELLI, A. PiCCININI, Il licenziamento individuale, Roma, 1991, 111-112; Cass. 8/7/88 n 4521 in Not. Giur. Lav., 1988, 725; Cass. 22/1/91 n542 in Giust. Civ., 1991, I, 1185 con nota di Poso Ancora sulle conseguenze del licenziamento disciplinare nelle piccole imprese. Altri ritenevano invece che a tale nullità corrispondesse solo l'obbligo del preavviso (Cass. 
Dunque, fino al 2012 il licenziamento disciplinare non preceduto dall'espletamento della procedura di cui all'art. 7 dello Statuto veniva assimilato al recesso ingiustificato con la conseguenza che ad esso veniva applicato il regime relativo alla dimensione del datore di lavoro.

Diversamente, la 1.92/2012 pur qualificando il licenziamento non preceduto da detta procedura come inefficace, lo ha sanzionato al nuovo sesto comma dell'art. 18 con la sola sanzione indennitaria dimezzata, senza alcun diritto alla conservazione del posto di lavoro.

Il d.lgs 23/2015 ha ridotto ulteriormente la sanzione parificandola a quella prevista per la mancata comunicazione dei motivi del recesso, vale a dire un'indennità non assoggettata a contribuzione previdenziale da un minimo di 2 ed un massimo di 12 mensilità ${ }^{20}$.

L'affievolimento delle tutele per i vizi formali della fase procedimentale del recesso deve essere letta unitamente al depotenziamento delle sanzioni per i vizi sostanziali del licenziamento, in quanto la tutela reintegratoria viene oggi garantita al lavoratore, oltre che per la mancanza di forma dell'atto, solo nelle ipotesi più gravi (mancanza di forma, discriminazione, lavoratrice in maternità, gravidanza e così via). In tale ottica, il vizio procedimentale impedisce o rende maggiormente difficoltoso al prestatore l'impugnazione del recesso che tuttavia lo porterebbe ad ottenere comunque esclusivamente una sanzione risarcitoria e non ripristinatoria del rapporto. In questo senso, la scelta di ridurre la sanzione per i vizi della procedura prevista per i licenziamenti collettivi e per quella dell'art. 7 1. 604/66 non appare in grado di indebolire irrazionalmente la posizione del lavoratore in quanto questi, in tali ipotesi, non potrebbe ottenere una tutela ripristinatoria del rapporto.

La violazione delle procedure di cui all'art. 7 dello Statuto appare tuttavia meno grave della mancata comunicazione dei motivi del recesso. In quest'ultimo caso, la motivazione consente al lavoratore un pieno diritto di difesa altrimenti certamente

4/3/93 n 2596 in Riv. Giur. Lav., 1993, II, 542; Cass. 27/3/85 n¹035 in Foro It., Rep. 1985, Voce Lavoro (Rapporto), 1974.). Altri ancora ritenevano ingiustificato il licenziamento intimato senza le prescritte formalità, con conseguente applicazione del regime relativo al numero di dipendenti occupati (Cass. 6/4/93 n ${ }^{\circ} 4131$ in Riv. Giur. Lav., 1993, II, 541; Cass. 24/2/93 n 2249 ivi, 543. Cass. 23/11/92 n 12486 in Dir. Prat. Lav., 1993, 713. ed in Riv. It. Dir. Lav., 1993, II, 355 con nota di MARINO. La prima ad argomentare in tal senso dopo la L. 108/90 é stata Cass 23/11/92 n 12486 cit., Pret. Milano, 22/11/93, Crescenzo c/Emme e E. S.r.l. in Riv. It. Dir. Lav., 1994, II, 545 con nota di MARINO che tende ad estendere anche al licenziamento orale tale teoria. Conf. TAZZI, op. cit., 435. Contra Cass. 542/91, cit.

20 La 1. 92/2012 aveva altresì introdotto una procedura di conciliazione preventiva per il recesso intimato per motivi economici al dichiarato scopo di trasformare il licenziamento da atto unilaterale dell'imprenditore in una scelta quanto più possibile condivisa, sul modello della procedura dei licenziamenti collettivi, al fine di ridurre il contenzioso che ne potrebbe derivare. Il mancato rispetto della procedura veniva sanzionato, sempre che il licenziamento non venga invalidato per altri motivi, con una sanzione compresa tra 6 e 12 mensilità dell'ultima retribuzione globale di fatto. Tale procedura non è stata tuttavia riproposta dal Legislatore con il d.lgs. 23/2015. 
menomato; il mancato rispetto delle garanzie procedimentali dell'articolo 7, e segnatamente la comunicazione preventiva delle contestazioni ed il diritto di presentare una propria difesa, non sembrerebbe violare il medesimo art. 24 Cost. giacché la fase pre-giudiziale di cui all'art. 7 Statuto non appare sorretta dalle medesime garanzie costituzionali che tutelano invece il diritto di agire in giudizio dopo il recesso.

Irrazionale appare piuttosto che per le sanzioni disciplinari non precedute dalla prevista procedura sia ancora applicabile la sanzione individuata da tempo dalla giurisprudenza vale a dire l'assoluta nullità del provvedimento disciplinare. Pertanto le sanzioni conservative sono ancora colpite da nullità laddove per la massima sanzione, quella espulsiva, viene prevista una conseguenza più lieve, con non pochi dubbi di ragionevolezza del sistema.

\section{LE CONSEGUENZE DELLE VIOLAZIONI DELLA PROCEDURA PREVISTA PER I LICENZIAMENTI COLLETTIVI}

La disciplina dei licenziamenti collettivi è stata oggetto di regolamentazione legislativa in Italia solo a partire dal 1991 quando, con la 1. 23/7/91 n.223, è stata recepita in Italia la direttiva C.E.E. 75/129; in precedenza, il mancato recepimento aveva provocato una serie di condanne del nostro Paese da parte della Corte di Giustizia $^{21}$.

La legge, nel disciplinare per la prima volta compiutamente i licenziamenti collettivi, ha predisposto una procedura di confronto tra le parti sociali con l'eventuale intervento dell'autorità pubblica che rappresenta uno degli aspetti maggiormente innovativi rispetto alla precedente regolamentazioni di origine patrizia, contenuta negli Accordi Interconfederali del 1950 e del $1965^{22}$.

E' opinione prevalente che, a seguito della legge, non esista più alcuna differenza tra licenziamento individuale o plurimo per ragioni oggettive e collettivo, in quanto è esclusivamente la portata degli interessi coinvolti, desunta dalla dimensione dell'impresa e dal numero dei licenziamenti, e l'allarme sociale che ne deriva a

21 Corte Giust. CEE 8/6/82 in FI, 1982, IV, 353 con nota di MAzzotTA; Corte Giust. CEE6/11/85 ivi, 1986, IV, 109 con nota di DE LuCA.

22 M. D'ANTONA, "Riduzione di personale e licenziamenti: la rivoluzione copernicana della 1.223/91" in Foro It., 1993, V, 2027ss; PIVETTI, "Alcune osservazione su licenziamenti collettivi e mobilità nella 1.223/91" in RGL, 1993, I, 51ss; L. MonTUSCHI, "Mobilità e licenziamenti: primi appunti ricostruttivi ed esegetici in margine alla L. $\mathrm{n}^{\circ} 223$ del 23/7/91" in Riv. It. Dir. Lav., 1991, I, 413; C. AlESSI, "Il licenziamento collettivo per riduzione di personale: fattispecie e disciplina" in Riv.Giur.Lav., 1995, I, 225; U. CARABELLI, "I licenziamenti per riduzione di personale" in $D L R I, 1994,213$; F. PATERNÒ, "Sulla nozione di licenziamento per riduzione di personale" in $A D L, 1995,2$, 77; Spagnuolo Vigorita, Guaglione, SCARPelli, "Commento all'art.24 della L.223/91" in Nuove Leggi Civ. Comm., 1994, 1091. Sulla procedura C. ZoLI, "La procedura" in QDLRI, 1997, 73ss. Sull'obbligo dell'imprenditore di trattare secondo correttezza e buona fede R. DEL PUNTA, "La L.223/91 e i licenziamenti collettivi: un primo bilancio" in QDLRI, 1997, 18. 
giustificare il ricorso ad una complessa procedura con il coinvolgimento sindacale ed amministrativo e l'applicabilità ai lavoratori coinvolti di strumenti di protezione sociale ${ }^{23}$.

In ossequio alla disciplina europea, la normativa italiana prevede all'art. 24 dei requisiti quantitativi e temporali per la qualificazione del licenziamento come collettivo: il datore di lavoro deve avere più di 15 addetti, deve effettuare 5 licenziamenti nell'arco di 120 giorni nell'ambito della stessa provincia e i licenziamenti dovranno essere "tutti riconducibili alla medesima riduzione o trasformazione" 24 . Rientrano pure nel campo di applicazione della normativa i licenziamenti per cessazione di attività, espressamente compresi dall'art.24 secondo comma.

La normativa si applica altresì ex art.4 ai licenziamenti intimati a lavoratori di imprese in Cassa Integrazione Guadagni Straordinaria, il cui ambito di applicazione è sensibilmente più ristretto rispetto ai limiti di cui all'art. 24, tranne che per il settore industriale; in tal caso, tuttavia, non è posto alcun limite al numero minimo di licenziamenti.

La normativa ha subito interventi successivi, tra cui va segnalato quello del decreto legislativo 8 aprile $2004, \mathrm{n}^{\circ} 110$ per adeguare, sotto il profilo dell'ambito soggettivo di applicazione, la norma alla direttiva $\mathrm{n}^{\circ} 98 / 59 / \mathrm{CE}^{25}$, estendendo l'ambito di applicazione a tutti i privati datori di lavoro compresi quelli non imprenditori.

Restano invece esclusi i licenziamenti per fine lavori edili, la cessazione di rapporti di lavoro a termine, compresi i dipendenti in prova.

Per quanto attiene al numero di 15 dipendenti, deve intendersi, ai sensi dell'art. 1, il computo dei dipendenti occupati mediamente nel semestre precedente ${ }^{26}$, da effettuarsi

23 In dottrina M. Miscione, "I licenziamenti per riduzione di personale e la mobilità" in F. CARINCI (a cura di) La disciplina dei licenziamenti dopo le leggi 108/90 e 223/91, Napoli, 1991, 299ss; O. MazzotTA, I licenziamenti, Milano, 1999, 640ss; R. Del Punta, La L.223/91 e i licenziamenti collettivi: un primo bilancio, cit., 15; M. D'ANTONA, Riduzione di personale e licenziamenti: la rivoluzione copernicana della L.223/91 cit, 2028. Già nella previgente normativa $c f r$. in dottrina F. MAZzIOTTI, Il licenziamento illegittimo, Napoli 1982, 169; G. PERA, La cessazione del rapporto di lavoro, Padova, 1980, 99; M. NAPOLI, La stabilità reale del rapporto di lavoro, Milano 1980. In giurisprudenza Cass. 26/4/96 n³896 in LG, 1996, 865; Cass. 218/86 n ${ }^{\circ}$ 538, in Riv. It. Dir. Lav., 1987, II, 593; in Dir. Prat. Lav., 1989, 1217. Contra, Per la tesi della distinzione ontologica Cass. 26/4/96 n³896 in Lav. Giur., 1996, 865.

24 R. Del Punta, "I licenziamenti collettivi" in Papaleoni, Del Punta, Mariani, La nuova Cassa integrazione guadagni e la mobilità, cit., 379. Sulla nozione di stabilimento nella direttiva Corte Giust. CE 1/2/07, causa C-270/05 in FI, 2007, IV, 270. Sulla non computabilità delle dimissioni incentivate nel numero dei rapporti cessati Cass. 23/6/06 n. 1463 in Riv. It. Dir. Lav., 2007, II, 425.

25 M .TATARELLI, "Estesa ai datori di lavoro non imprenditori la procedura sui licenziamenti collettivi", in Guida Lav., 2004, 20, 32.

26 In tal senso anche la circolare ministeriale 155/91, in DPL, 1991, 3321. 
al momento dell'avvio della procedura, mentre l'ambito nel quale calcolare il numero dei dipendenti dell'impresa è l'intero complesso aziendale sul territorio nazionale. La procedura sindacale con il controllo amministrativo prevede che il datore che voglia procedere a riduzioni di personale o messa in mobilità sia obbligato a comunicare per iscritto agli organismi sindacali indicati dalla legge tale intenzione specificando una serie di informazioni previste dal terzo comma dell'art. $4^{27}$.

La comunicazione deve contenere i motivi che hanno determinato la situazione di eccedenza, i motivi tecnici organizzativi e produttivi a fronte dei quali appaiono inattuabili altri rimedi idonei ad evitare i licenziamenti, ed infine numero, collocazione aziendale e profili professionali del personale eccedentario nonché i tempi di attuazione del programma e le misure programmate per evitare le conseguenze sociali dei licenziamenti. Tale contenuto è stato ulteriormente ampliato dalla 1.151/97 con l'introduzione dell'obbligo di comunicare il personale normalmente occupato ed il metodo di calcolo degli incentivi ed integrazioni al reddito aggiuntivi rispetto a quelli legali, oltre all'obbligo di fornire le informazioni richieste anche in caso di procedura posta in essere da impresa controllata qualora l'avvio della procedura sia stata decisa dall'impresa controllante (art. 4 comma 15 bis). Lo scopo di tale procedura è quello di fornire tutte le informazioni necessarie al sindacato per valutare le azioni da intraprendere ${ }^{28}$.

Successivamente, su richiesta delle organizzazioni sindacali, si procede ad un esame congiunto prima con il solo sindacato e, in caso di mancato accordo, con l'intervento della Direzione territoriale del lavoro.

Nel caso in cui non vengano raggiunti accordi in grado di evitare del tutto o in parte $\mathrm{i}$ licenziamenti, il datore ha facoltà di procedere ai recessi comunicandoli per iscritto a ciascun lavoratore nel rispetto dei termini del preavviso, inviando contestualmente all'autorità amministrativa e alle organizzazioni sindacali oggetto della prima comunicazione gli elenchi del personale licenziato completi dei dati anagrafici e familiari e delle modalità applicative dei criteri di scelta (art.4 nono comma).

La finalità della comunicazione iniziale alle organizzazioni sindacali è facilmente individuabile nella necessità di porre queste ultime a conoscenza della situazione che ha determinato la necessità di licenziare il personale onde poter valutare se intervenire o meno, con quali strumenti e se addivenire o meno ad un accordo ed a quali condizioni. La ratio della comunicazione finale di cui al nono comma è invece più complessa, nel senso che essa appare diversa a seconda degli adempimenti previsti dalla norma e dei soggetti destinatari della stessa. La comunicazione delle modalità applicative è infatti un atto che deve precedere quella dei recessi, giacché logicamente il datore deve prima elaborarle e poi applicarle al caso concreto, individuando i singoli

\footnotetext{
27 Sui soggetti destinatari delle comunicazioni Cass. 14/1/05 n.639 in Riv. It. Dir. Lav., 2005, II, 950; Cass. 24/3/04 n. 5942 ivi, II, 657.

28 Sul momento iniziale della procedura Corte Giust. 27/1/05 c.188/03 in Mass. Giur. Lav., 2005, 385; in Foro It., 2005, 185; in Guida Lav., 2005, 10, 32.
} 
lavoratori da licenziare: è per tale motivo che le due comunicazioni devono avvenire contestualmente ${ }^{29}$.

L'art. 5 della legge in esame individua dei criteri di scelta dei lavoratori da licenziare e precisamente $\mathrm{i}$ criteri tecnico produttivi ed organizzativi, i carichi di famiglia e l'anzianità del lavoratore. Essi sono però sussidiari rispetto ad altri eventuali criteri che le parti abbiano concordato tra loro; in tal caso, gli accordi che prevedono i criteri di scelta hanno natura gestionale e si applicano a tutti i lavoratori, iscritti o meno alle organizzazioni stipulanti ${ }^{30}$.

Diversamente dai licenziamenti individuali, il lavoratore non può richiedere al datore di lavoro i motivi del recesso né gli atti della procedura. Va segnalato tuttavia come tale impostazione appaia gravemente lesiva del diritto di difesa del lavoratore il quale, soprattutto qualora non iscritto ad uno dei sindacati firmatari degli accordi, può non essere posto in condizioni di consultare preventivamente la procedura ed i criteri di scelta per valutarne la correttezza, pur restando obbligato nei sessanta giorni dal recesso ad impugnarlo ${ }^{31}$.

Anche la disciplina dei licenziamenti collettivi, ed in particolare la procedura di consultazione, è stata modificata dalla 1. 92 del 2012. In dettaglio, la novella ha previsto la possibilità per l'imprenditore di inviare la comunicazione finale non più necessariamente contestualmente ai licenziamenti degli elementi previsti dall'art. 4 comma nono; delle modifiche al regime sanzionatorio, l'efficacia sanante di eventuali vizi della procedura dell'accordo raggiunto in sede sindacale e l'applicabilità del nuovo termine di decadenza previsto per i licenziamenti individuali.

La previsione in base alla quale il raggiungimento di un accordo ha effetto sanante di eventuali vizi della procedura recepisce fondamentalmente un'interpretazione già fatta propria dalla giurisprudenza maggioritaria, che aveva riconosciuto tale possibilità alle parti nel corso della procedura. In precedenza, la violazione della procedura era sanzionata con l'inefficacia dei licenziamenti, mentre il mancato rispetto dei criteri di scelta comportava l'annullabilità dei recessi, in ogni caso con la sanzione della reintegrazione di cui all'art. $18 \mathrm{St}$.

Le modificazioni operate al regime sanzionatorio dalla riforma del 2012 rispondono invece all'esigenza di raccordare le novità in tema di sanzioni per i licenziamenti ai vizi dei licenziamenti collettivi. Mentre in caso di violazione dei criteri di scelta si applicherà la reintegrazione con risarcimento del danno forfettizzato, viene previsto

29 Cass. 8/3/06 n. 4970 in Mass. Giur. Lav., 2006, 778; in Riv. It. Dir. Lav., 2007, II, 163; Cass. 7/12/07 n. 25668 in Guida Dir., 2008, 3, 63; Cass. 28/7/05 n.15898, in Mass. Giur. Lav., 2006, 141 con nota di TOMASSINI.

30 Corte Cost. 22/6/94 n.268 in Foro It., 1994, 2307; in Mass. Giur. Lav., 1994, 473 con nota di ManNACCIO; in Dir. Lav., 1994, II, 160 con nota di N. BeTtinI.

31 Sulla necessità che il lavoratore indichi le specifiche omissioni ed irregolarità della procedura Cass. 8/8/05 n. 16629 in Mass. Giur. Lav., 2006, 192. Per la carenza di interesse ad agire del lavoratore per segnalare l'incompletezza della procedura che non abbia avuto conseguenze sul proprio licenziamento Cass. 11/1/08 n. 528 in Guida Dir., 2008, 7, 22. 
un ridimensionamento della tutela accordata in caso di violazione della procedura. In particolare, nel caso del licenziamento collettivo si applica invece la tutela risarcitoria forte da 12 a 24 mensilità. In tal modo, sebbene la tutela non sia più la reintegrazione, che si applicherà solo per la violazione dei criteri di scelta, appare evidente che il Legislatore continua a ritenere il rispetto della procedura più rilevante nel licenziamento collettivo che nel licenziamento individuale, dove la violazione degli aspetti procedurali comporta la tutela risarcitoria debole, da 6 a 12 mensilità ${ }^{32}$.

Con la riforma del 2015, invece, la tutela reintegratoria viene del tutto eliminata unificandosi la sanzione prevista in caso di violazione delle procedure richiamate all'articolo 4, comma 12, con quella dei criteri di scelta di cui all'art. 5, comma 1, della legge n. 223 del 1991, applicando per entrambe il regime risarcitorio nella forma forfettizzata in relazione all'anzianità di servizio e alle dimensioni dell'impresa prevista dal Jobs act innanzi vista ${ }^{33}$.

Appare evidente come soprattutto questa modificazione possa comportare per i lavoratori rischi di abusi nella fase di individuazione dei lavoratori da licenziare.

In definitiva, con la riforma del 2015, per i licenziamenti per ragioni economiche, siano essi individuali o collettivi, viene del tutto meno la possibilità di ottenere la reintegrazione anche nel caso in cui si dimostrasse l'assoluta inesistenza della ragione oggettiva posta a base del recesso, come nell'ipotesi in cui venissero omesse le garanzie procedurali.

\section{CONCLUSIONI}

Il depotenziamento delle sanzioni operato prima dalla 1. 92/2012 quindi dal d.lgs 23/2015 per la violazione delle garanzie di forma e procedura costituisce un profilo estremamente delicato in grado di ledere in maniera sensibile il diritto di difesa del prestatore di lavoro.

La riduzione delle sanzioni per la procedura del licenziamento disciplinare, in particolare, sarebbe meno grave se non si aggiungesse al depotenziamento delle sanzioni previste per la mancata comunicazione delle motivazioni, in quanto esporrebbe il lavoratore esclusivamente alla necessità di attendere il momento espulsivo per conoscere le ragioni dell'atto e valutare se impugnarle o meno, facendo valere in quella sede le proprie difese. Diversamente, il prestatore potrebbe oggi trovarsi di fronte ad un licenziamento disciplinare del quale ignori non solo le

32 P. LAmBERTUCCI, La disciplina dei licenziamenti collettivi nella legge 28 giugno 2012 n. 92 in materia di riforma del mercato del lavoro: prime riflessioni in Arg. Dir. Lav, 2013, 242ss; L. LOREA, Riduzione di personale e articolazione della disciplina del licenziamento: le tutele procedurali in Dir. Mer. Lav., 2012, 487ss.

33 In particolare, una o due mensilità per ciascun anno di servizio in relazione alla dimensione dell'impresa fino ad un massimo di dodici anni, quindi un importo pari al più a 12 o 24 mensilità. 
specifiche motivazioni ma perfino la natura stessa del recesso, che potrebbe essere privo di qualunque indicazione.

Diviene quindi fondamentale l'articolazione dell'onere della prova in quanto il lavoratore, al quale non è stato consentito di conoscere preventivamente le ragioni del recesso e che non può richiedere nuovi mezzi di prova dopo il deposito del ricorso, trova unica e fondamentale garanzia nella circostanza che l'onere della prova delle ragioni del licenziamento spetta ex art. 6 1.604/66 al datore di lavoro. La soluzione che può quindi prospettarsi onde evitare eventuali vizi di incostituzionalità della norma è quella di consentire al lavoratore di integrare le ragioni del ricorso una volta che il datore di lavoro abbia esplicitato le motivazioni del ricorso del recesso per la prima volta nella comparsa di costituzione.

Un'ulteriore diversa soluzione potrebbe essere quella di considerare la tempestività (e quindi completezza) della contestazione quale elemento costitutivo del diritto di recesso con conseguente inesistenza giuridica del fatto contestato e conseguente applicabilità del regime reintegratorio del quinto comma dell'art. $18^{34}$.

Può notarsi come una situazione simile si verifica per i licenziamenti collettivi dove pure non è previsto l'invio al prestatore di lavoro degli atti della procedura; conseguentemente, il lavoratore che non sia iscritto ad un sindacato coinvolto nella consultazione non è in grado di conoscere la procedura stessa e, quindi, di individuarne eventuali vizi.

In questo caso, tuttavia, la rimodulazione delle sanzioni previste sia sul piano sostanziale sia su quello formale finiscono col rendere meno rilevante l'individuazione della funzione, precedentemente essenziale, della procedura. In particolare, la comunicazione al termine della procedura delle modalità di applicazione dei criteri di scelta alle OO.SS nelle comunicazioni finali ha il fine evidente di consentire al Sindacato un accurato controllo sulla corretta individuazione dei nominativi da licenziare ma finisce con il perdere rilievo nel momento in cui un'applicazione inesatta, se non arbitraria, dei criteri non viene sanzionata con la reintegrazione ma con il mero risarcimento del danno. Diversamente, nel caso del licenziamento disciplinare, quando il fatto materiale sia insussistente al lavoratore spetterebbe la reintegrazione, e dunque la violazione appare in grado di ledere irrimediabilmente i diritti del prestatore licenziato.

\section{BIBLIOGRAFIA}

AA.VV., La riforma del lavoro (c.d. Jobs Act). Il contratto di lavoro a tutele crescenti e gli strumenti di contrasto alla disoccupazione (d.leg. 4 marzo 2015 nn. 22 e 23), in Foro It., 2015, V, col. 229.

34 Cass. 6/11/14 n. 23669 cit. In dottrina A. TAMPIERI, "I vizi formali del licenziamento" in G. ZiLIO GRANDI, M. BIASI, Commentario breve alla riforma Jobs Act cit., 254 ed ivi per ulteriori riferimenti. 
Alessi C., "Il licenziamento collettivo per riduzione di personale: fattispecie e disciplina" in Riv. Giur. Lav., 1995, I, 225.

Ballestrero M.V., I licenziamenti, Milano, 1975, 276.

Balletti E., "Riassunzione o risarcimento del danno" in I licenziamenti individuali a cura di G. Ferraro, Napoli 1990, 93.

Carabelli U., "I licenziamenti per riduzione di personale" in DLRI, 1994, 213.

Carinci F., "Un contratto alla ricerca di una sua identità: il contratto a tempo indeterminato a tutele crescenti (ai sensi della bozza del decreto legislativo 24 dicembre 2014)", in F. Carinci, M. Tiraboschi (a cura di), I decreti attuativi del Jobs Act: prima lettura e interpretazioni, Modena, 2015, 65ss.

Carinci F., Cester C. (a cura di), Il licenziamento all'indomani del d.lgs. n. 23/2015 (contratto di lavoro a tempo indeterminato a tutele crescenti), Modena, 2015.

D'antona M., "Riduzione di personale e licenziamenti: la rivoluzione copernicana della l.223/91" in Foro It., 1993, V, 2027ss.

D'onghia M., Bavaro V., "Profili costituzionali del licenziamento nullo" in WP C.S.D.L.E. “Massimo D'Antona”.IT, 2016, 305, 17ss.

D'onghia M., La forma vincolata nel diritto del lavoro, Milano, 2005, 85ss.

De R. Luca Tamajo, "Disciplina dei licenziamenti individuali" in Le nuove leggi civili commentate, a cura di R. De Luca Tamajo, M. D’Antona, 1991, 187.

De Luca Tamajo R., F. Bianchi D’Urso, “Licenziamento disciplinare e Corte costituzionale”, in Not. Giur. Lav., 1989, 148.

Del Punta R., "La L.223/91 e i licenziamenti collettivi: un primo bilancio" in QDLRI, 1997, 18.

Di Majo A., "I licenziamenti illegittimi tra diritto comune e diritto speciale" in Riv. Giur. Lav., 1974, I, 272.

Ferraro G., "Formalismo giuridico e diritto del lavoro", in Dir. Lav. Rel. Ind., 1989, 555.

Ferri G.B., "Forma e contenuto degli atti giuridici", in Riv. Dir. Comm., 1990, I, 1.

Fiorillo L., Perulli A., Contratto a tutele crescenti e Naspi, Torino 2015.

Focareta F., "L'articolo 18 St. lav. fra tendenze espansive e problemi irrisolti", in Quad. Dir. Lav. Rel. Ind., 1989, 6, 269.

Garofalo M. G., “La nuova disciplina dei licenziamenti individuali: prime osservazioni”, in Riv. Giur. Lav., 1990, I, 184.

Genoviva P., I licenziamenti, Torino 1988.

Ghera E., Garofalo D. (a cura di), Le tutele per i licenziamenti e per la disoccupazione involontaria nel Jobs Act 2, Bari, 2015.

Gianpiccolo G., "Dichiarazione recettizia”, in Enciclopedia del diritto, XII, Milano 1964, 389.

Giorgianni M., "Forma degli atti (diritto privato)”, in Enciclopedia del diritto, XVII, 1968, Milano 1968, 993.

Ichino P., "La riforma del lavoro in Italia. Una nuova cultura delle relazioni industriali", in Riv. It. Dir. Lav., 2015, I, 205ss.

Lambertucci P., La disciplina dei licenziamenti collettivi nella legge 28 giugno 2012 n. 92 in materia di riforma del mercato del lavoro: prime riflessioni in Arg. Dir. Lav, 2013, 242ss.

Marazza M., "Il regime sanzionatorio dei licenziamenti nel Jobs Act”, in Arg. Dir. Lav., 2015, 2, 310ss. 
Marino, "Il vizio di forma del licenziamento viziato nella procedura: una svolta di rilievo", in Riv. It. Dir. Lav., 1993, II, 357ss.

Mazziotti F., "Forma e procedura dei licenziamenti" in F. Carinci, La disciplina dei licenziamenti dopo le leggi 108/1990 e 223/1991, Napoli 1991, 71.

Mazziotti F., "Licenziamenti illegittimi e provvedimenti giudiziari", in Atti delle giornate di studio di diritto del lavoro, Torino, 16-17 maggio 1987, Milano, 1988, 162.

Mazziotti F., Il licenziamento illegittimo, Napoli 1982.

Mazzotta O., I licenziamenti, Milano, 1999.

Miscione M., "I licenziamenti per riduzione di personale e la mobilità" in F. Carinci (a cura di) La disciplina dei licenziamenti dopo le leggi 108/90 e 223/91, Napoli, 1991, 299ss.

Montuschi L., "Mobilità e licenziamenti: primi appunti ricostruttivi ed esegetici in margine alla L. $n^{\circ} 223$ del 23/7/91" in Riv. It. Dir. Lav., 1991, I, 413.

Napoletano G., La nuova disciplina dei licenziamenti individuali, Napoli 1990, 97.

Napoli M., La stabilità reale del rapporto di lavoro, Milano 1980. In giurisprudenza Cass. 26/4/96 n³896 in $L G, 1996,865$.

Niccolai, “Gli effetti del licenziamento individuale illegittimo" in Lav. Dir., 1982, 119.

Nogler L., "I licenziamenti per giustificato motivo soggettivo o per giusta causa nel d.lgs. $n .23$ del 2015", in Arg. Dir. Lav., 2015, 3, 507ss.

Papaleoni M., "Licenziamento disciplinare e nullità di diritto comune" in Riv. It. Dir. Lav., 1995, II, 653.

Papaleoni M., La fine del libero licenziamento, Milano, 1991.

Pera G., Ancora sul vizio di forma nella zona della tutela debole contro il licenziamento in Giust. Civ., 1997, I, 938.

Pera G., La cessazione del rapporto di lavoro, Padova, 1980.

Pera G., Le novità nella disciplina dei licenziamenti, Padova, 1993.

Perlingieri P., "La forma legale del licenziamento individuale come "garanzia"” in Rass. Dir. Civ., 1986, 1071.

Perlingieri P., Forma degli atti e formalismo degli interpreti, Napoli, 1987.

Persiani M., "Noterelle su due problemi di interpretazione della nuova disciplina dei licenziamenti", Arg. Dir. Lav., 2015, 393ss.

Pessi R., Pisani C., Proia G., Vallebona A. (a cura di), Jobs Act e licenziamento, Torino, 2015.

Pivetti, "Alcune osservazione su licenziamenti collettivi e mobilità nella l.223/91" in RGL, 1993, I, 51ss.

Roccella M., "I licenziamenti nella piccola impresa” in Dir. Prat. Lav., 1990, 666.

Romagnoli U., "Sub. art. 18" in Ghezzi, Mancini, Montuschi, Romagnoli, Statuto dei diritti dei lavoratori, Bologna - Roma, 1972, 95.

Sacco R., Il contratto, Torino 1993.

Scalisi V., "Inefficacia" in Enciclopedia del diritto, 1971, 325.

Spagnuolo Vigorita, Guaglione, Scarpelli, "Commento all'art.24 della L.223/91" in Nuove Leggi Civ. Comm., 1994, 1091.

Tatarelli M., "Estesa ai datori di lavoro non imprenditori la procedura sui licenziamenti collettivi", in Guida Lav., 2004, 20, 32. 
Tullini P., "La cd. tutela reale di diritto comune", in Quad. Dir. Lav. Rel. Ind., n. 8, 1990, 103 ss.

Vidiri G., "Il licenziamento disciplinare nel primo decreto attuativo del Jobs Act tra luci e (non poche) ombre", in Arg. Dir. Lav., 2015, 353ss.

Zilio Grandi G., Biasi M. (a cura di), Commentario breve alla riforma “Jobs act”, Padova, 2016.

Ziliotti M., "Commento all'art.2" in L. Galantino, La disciplina dei licenziamenti individuali e collettivi, Torino, 1993, 22. 